\title{
Avanço e consequências da modernidade global*
}

\section{Volker H. Schmidt**}

Resumo: Este trabalho propõe o conceito de modernidade global a partir da observação de dados mundiais. O conceito busca oferecer uma perspectiva integrativa que considera múltiplas dimensões da vida, a partir de no mínimo quatro dimensões inter-relacionadas: indivíduo, cultura, organismo e sistemas sociais.

Por meio de uma perspectiva histórica da economia, e questões como a redução da pobreza, a ascensão da cidade, a educação formal, a ciência e a academia, dentre outros diversos campos, constrói-se o debate sobre o conceito, ilustrado pelo desenvolvimento moderno e sua velocidade. Ao comparar as mudanças ocorridas em décadas recentes aos séculos anteriores, o texto sugere que a modernidade global pode ser considerada a ferramenta sociológica utilizada para elucidar a rapidez em que essas mudanças ocorrem, bem como suas dimensões e consequências; o que termina por ilustrar o contexto da sociedade mundial.

Palavras-chave: Sociologia. Modernidade global. Contemporaneidade. Sistemas Sociais. Novos centros de poder da modernidade.

\section{Breakthrough and consequences of global modernity}

Abstract: This work puts forward the concept of global modernity starting from the observation of worldwide data. This concept seeks to provide an integrative perspective that takes multiple dimensions of life into consideration, on the basis of at least four interrelated dimensions: the individual, culture, the organism and social systems.

The discussion of the concept is elaborated through a historical perspective on economy, as well as the examination of issues such as poverty reduction, the rise of the city, formal education, science and academia, among a variety of other fields, and illustrated by modern development and its speed. Comparing the changes that have occurred in recent decades to previous centuries, the text suggests that global modernity can be considered a sociological tool used to explicate the speed at which these changes occur, as well as their dimensions and consequences - thereby illustrating the context of global society.

Keywords: Sociology. Global modernity. Contemporaneity. Social systems. New power centers of modernity.

\author{
* Tradução: Léo \\ Lopes e Solange \\ Miguel Marcondes \\ Armando. \\ ** Volker $\mathrm{H}$. \\ Schmidt é professor \\ de sociologia na \\ National University \\ of Singapore (NUS), \\ Singapura; também \\ já lecionou nas \\ universidades de \\ Mannheim, e de \\ Bremen, ambas \\ na Alemanha, e \\ participou das \\ atividades do J. F. \\ Kennedy Memorial \\ Fellow do Centro de \\ Estudos Europeus \\ da Universidade de \\ Harvard, Cambridge, \\ Massachusetts, \\ Estados Unidos. \\ <socvhs@nus.edu. \\ sg>.
}


s últimas quatro décadas, aproximadamente, testemunharam o que provavelmente equivale à maior e mais dramática transformação da história humana. Durante esse período, vários limites críticos foram trespassados, levando muitos dos marcos do desenvolvimento moderno a alcançar pela primeira vez proporções genuinamente globais; moldando as vidas das pessoas; e transformando o que até então era um fenômeno minoritário em uma realidade vivenciada pela maioria da população humana (Schmidt, 2007). Para entendermos este desenvolvimento, proponho o conceito de modernidade global. Esse conceito agrupa e sistematiza, em um nível de abstração relativamente elevado, mudanças que foram observadas separadamente na literatura das ciências sociais pertinente, incluindo a literatura sobre globalização, que aumentou consideravelmente nossa compreensão do mundo social contemporâneo, mas, ao mesmo tempo, carece de uma perspectiva integrativa. O conceito de modernidade global, precisamente, visa fornecer tal perspectiva, tratando a mudança como processo altamente complexo, multidimensional, e, ainda assim, inter-relacionado, que não deixa intocada nenhuma esfera da vida.

O trabalho se desenvolve da seguinte forma: em uma primeira etapa (Seção II), apresentarei dados de vários campos para ilustrar o que quero dizer com o avanço global da modernidade. Farei, então, um breve esboço do conceito de modernidade global tal como o utilizo (Seção III). Por fim, farei duas sugestões quanto a prováveis consequências deste avanço (Seção IV).

A começar pela economia, o mundo de hoje está mais rico do que poderia antever o mais perspicaz dos observadores do século XIX, e muito dessa riqueza é produto do crescimento econômico moderno, que se acredita ter irrompido por volta de 1820 (ver Maddison, 2001). Após esta decolagem, o valor do PIB mundial cresceu o equivalente a US\$ 22 trilhões até 1985. Nada disso havia acontecido antes. Mas, por mais espetacular que possa parecer a qualquer contemporâneo historicamente consciente, pelos padrões de hoje, esse "milagre econômico" é, de fato, bastante inexpressivo, pois, já em 2010, apenas 25 anos depois, o PIB global havia aumentado em outros US\$ 30 trilhões (Eckes, 2011). Assim, o valor adicionado em um único quarto de século é substancialmente maior do que o somatório dos 150 anos anteriores. Uma comparação com estágios anteriores da evolução da sociedade nos dá uma ideia ainda mais clara do quão fundamental é essa mudança. Por 
exemplo, estima-se que o crescimento econômico nos três anos de 1995 a 1998 seja "maior que o crescimento total nos 10 mil anos anteriores a 1900" (Christian, 2004: 446). Não surpreendentemente, esse padrão também se traduz em rendas mais altas. Medida em dólares constantes e utilizando-se a paridade de poder de compra (PPC), a média mundial das rendas per capita aumentou em US\$ 5.000 entre 1870 e 1980 (de US\$ 870 para US\$ 5.949 ao ano). Esse é um desenvolvimento notável. Mas então elas dispararam mais US\$ 3.500 nas três décadas seguintes (para US\$ 9.541 em 2009; cf. Eckes, 2011). Se confiarmos nos dados apresentados pela CIA (2017), elas agora estão em US\$ 16.300, medidas em dólares de 2016.

O crescimento econômico não é um fenômeno isolado, é acompanhado por desenvolvimentos em escala semelhante em outras esferas, algumas das quais abordarei na sequência. Mas primeiro vou me ater um pouco mais ao campo econômico. Como todos sabemos - e muitos de nós lamentamos - os frutos do crescimento são distribuídos de forma muito desigual. Se fossem distribuídos de forma mais igualitária, muito mais pessoas poderiam se beneficiar e ninguém sofreria de pobreza. No mundo pré-moderno, a pobreza era em grande parte um problema de produção - a soma do produto social era simplesmente pequena demais para elevar todos acima da linha da pobreza, significando que a pobreza era a expectativa "normal" para a maioria dos setores da população. Hoje a pobreza é um problema de distribuição; os recursos necessários para erradicá-la estão, em princípio, disponíveis. Dado este cenário, a continuação da existência da pobreza em massa é amplamente vista como um escândalo. No entanto, reduções significativas na população global de pobres de fato ocorreram ao longo dos últimos 200 anos. Usando o padrão de consumo de um dólar por dia do Banco Mundial para determinar a pobreza absoluta, três quartos dos nossos antepassados recaíam abaixo dessa linha até 1820, e, com uma renda per capita estimada de US\$ 651 ao ano, o dito "cidadão" médio do mundo estava de fato muito próximo disso (Firebaugh, 2003: 13). Passados 160 anos, em 1981, a maioria da população mundial (51\%) ainda era pobre segundo o padrão, um pouco mais exigente, de US\$1,25 por dia, usado até recentemente pelo Banco Mundial (UNDP 2014: 19). Depois de reajustar o padrão para US\$1,90 por dia, em 2015, o Banco Mundial estima que a população ao nível da pobreza extrema se reduziu a menos de $10 \%$ da população mundial, para 705 milhões de pessoas, abaixo dos 2,2 bilhões de meio século antes (Roser \& Ortiz-Ospina, 2017). Isso ainda reflete muito sofrimento desnecessário, contudo significa que mais de $90 \%$ das pessoas em todo o mundo escaparam das piores formas de pobreza e, embora os números fossem diferentes, a tendência seria a mesma se elevássemos a linha de pobreza a um padrão mais elevado. A recente ascensão de uma classe média genuinamente global (ver, por exemplo, Kharas, 2010) espelha essa tendência. 
Três quartos dos pobres - restantes - vivem do campo. Durante a Era Neolítica, a esmagadora maioria da humanidade garantia seu sustento através da agricultura. Isso começou a mudar com a Revolução Industrial, cujo impacto inicialmente permaneceu pequeno, mesmo na Grã-Bretanha, onde afetou apenas um setor relativamente pequeno da economia até o século XIX. Nas décadas de 1930 e 1940, a população agrícola ainda compreendia até $40 \%$ da população em muitos dos países mais avançados do ponto de vista socioeconômico do mundo, numa queda de 60 a $90 \%$ dos séculos anteriores à Revolução Industrial (Crone, 1989). Na década de 1980, ela havia sido reduzida a níveis entre três e cinco por cento. Assim, em questão de aproximadamente 200 anos, o que determinou as vidas da esmagadora maioria da humanidade durante milênios praticamente desapareceu desta parte do planeta. Em outras regiões, onde o campesinato se estabeleceu mais tarde, o seu declínio foi ainda mais rápido. Até 1950, 64\% da força de trabalho global ainda estava engajada na agricultura. A mudança final em direção a uma força de trabalho predominantemente não agrícola ocorreu depois de 1980, quando a parcela de camponeses caiu pela primeira vez abaixo dos 50\%, de 52 para 47\% em 1990 (FAO 2000: 18). Hoje, a composição setorial da força de trabalho global está se aproximando do padrão que vigorou no Ocidente em várias décadas passadas, com o maior grupo de empregados $(44,7 \%)$ trabalhando em serviços, apenas $31,7 \%$ na agricultura e os outros $23,6 \%$ sendo trabalhadores industriais (CIA, 2017).

Uma mudança que normalmente acompanha o declínio do campesinato é a ascensão da cidade. A vida moderna - geralmente se concorda - é vida urbana. Mas até recentemente, a maioria da população mundial vivia em áreas rurais. Isso não é mais o caso. Entre 2007 e 2010, metade da população mundial tornou-se urbana pela primeira vez, compreendendo 54\% em 2015. As cidades existem há milênios, mas o processo de urbanização propriamente moderno foi bastante gradual e não uniforme. Em 1820, apenas 2,5\% da população mundial vivia em cidades com mais de 20 mil habitantes (Kumar, 1999). Em 1900, esse número subiu para 13\% (Economist, 3 Maio 2007) -, quintuplicou em 80 anos, mas em termos de seus efeitos sobre a humanidade ainda está muito distante dos desenvolvimentos que se seguiram à Segunda Guerra Mundial. Assim, entre 1950 e 1980, a população urbana mundial cresceu em um bilhão de pessoas, e mesmo esse aumento parece pouco em comparação ao período mais recente: entre 1980 e 2010, quando essa população cresceu mais 1,75 bilhão, ultrapassando por fim a ainda considerável população rural, que deverá diminuir ainda mais nas próximas décadas (UN, 2014).

Tais tendências levam a um aumento dramático na expectativa de vida global, que atualmente é de 69 anos no nascimento (CIA, 2017). É exatamente isso que uma pessoa nascida em 1960 em um país de alta renda poderia esperar, e excede em 
mais de $50 \%$ a faixa de 41 a 45 anos típica dos que compunham os países socioeconômicos mais avançados da Europa Ocidental e da América do Norte no final do século XIX (Easterlin, 2000; Eckes, 2011). De fato, até mesmo a África Subsaariana registra, em grande parte, expectativas de vida de 50 anos ou mais agora, com apenas alguns países pairando em torno dos 45 anos, marca que os principais países ocidentais alcançaram na virada para o século XX (UNDP, 2014).

A educação formal é uma das principais características do desenvolvimento moderno, mas a inclusão massiva da população global é um fenômeno recente. Em 1950, $53 \%$ dos adultos com 15 anos ou mais obtiveram pelo menos alguma escolarização, mas demorou até a década de 1970 para que o nível de analfabetismo caísse para menos de 50\%; hoje, aproximadamente $86 \%$ da população mundial pode pelo menos ler e escrever. Em 2010, quase todas as crianças em idade de educação primária estavam matriculadas, e $86 \%$ dos adultos tiveram alguma educação formal, com cerca de $50 \%$ expostos a vários anos de educação secundária também. A média de anos de escolarização passou de 3,17 para 7,76, neste período. Os números absolutos são ainda mais impressionantes, tanto por si quanto em termos de suas implicações para a formação do capital humano. Em 1960, 150 milhões de adultos em todo o mundo haviam completado pelo menos o ensino médio, dos quais 80 milhões viviam no mundo desenvolvido. Hoje, existem 1,24 bilhão, dos quais 940 milhões estão nos países em desenvolvimento (Barro \& Lee, 2013). O ensino superior mostra um aumento ainda mais acentuado. Em 1900, cerca de 500 mil estudantes estavam matriculados em organizações de ensino superior em todo o mundo, representando 1\% das pessoas em idade universitária. Em 2000, o número cresceu duzentas vezes, alcançando 100 milhões de matrículas, o que representa cerca de $20 \%$ do coorte global (Schofer \& Meyer, 2005). Apenas uma década após o milênio, outro aumento de 80\% ocorreu, com 182 milhões de estudantes matriculados globalmente até 2011 (Unesco, 2014: 16). Assim, uma única década acrescenta quase tanto crescimento absoluto quanto todo o século anterior.

Não menos decisiva é a expansão da ciência. A atual revolução acadêmica é muito mais ampla e provavelmente mais consequente do que aquela que criou a universidade de pesquisa, em meados do século XIX. Na virada do século XX, as universidades alemãs, geralmente consideradas como tendo liderado o mundo da ciência na época, tinham, entre elas, 2.667 acadêmicos (Cozzens, 1997). Em 2015, esse número subiu para 239.200, e o forte contingente de 46.344 professores alemães superam, sozinhos, em várias vezes, toda a força de trabalho acadêmica de 100 anos atrás (Statistisches Bundesamt, 2016: 24). Globalmente, o número de cientistas pesquisadores (excluindo ciências sociais e humanas) aumentou de 5,2 miIhões em 1997 para 7,8 milhões uma década e meia depois (Unesco, 2001; 2015). 
A arrancada na produção científica é ainda mais imponente. Após vários séculos de crescimento (Price, 1963), a ciência global atingiu um ponto de inflexão após o qual os números se tornaram incrivelmente elevados. Assim, aproximadamente 360 mil artigos de ciência e engenharia foram publicados anualmente, até 1985. Então, em questão de menos de duas décadas, esse número subiu para 1,1 milhão por ano em 2003. Apenas dez anos depois, em 2014, alcançou quase 2,2 milhões (veja o quadro no World Bank), e em sua taxa de crescimento atual de 7 a $9 \%$ (Bornemann \& Mutz, 2015; National Science Board, 2016) alcançará 4,5 milhões na próxima década. As tendências registro de patentes exibem uma trajetória similar. Em ambos os casos, a principal força motriz é a recente acumulação maciça de capacidades de pesquisa na Ásia Oriental (veja Wipo, 2014; Unesco, 2015; Hu et alii, 2017).

A lista de mudanças não para por aqui. Ela poderia ser aumentada por vários desenvolvimentos importantes nos campos da tecnologia (e.g. a rápida expansão dos sistemas de transporte de alta velocidade e de comunicação de massa que aumentaram enormemente a mobilidade, a conectividade e a consciência mundial de bilhões de pessoas em poucas décadas), a economia global (ou seja, a reversão de uma situação em que apenas uma minoria da população vivia sob instituições capitalistas para a situação atual onde isso é a realidade para a grande maioria), no campo político (desde 1992, mais da metade de todos os países foi governada democraticamente pela primeira vez), e assim por diante.

Considerados individualmente, cada um dos desenvolvimentos acima marca uma mudança radical no(s) domínio(s) da vida e setor(es) da sociedade que afetam. Juntos, eles equivalem a nada menos que uma revolução social - o avanço definitivo da modernidade em escala global. Temporalmente, a maior parte dessa revolução está concentrada na última parte do século XX; espacialmente, concentra-se na Ásia, especialmente na Ásia Ocidental, com o sul da Ásia "juntando-se a ela" um pouco mais tarde, mas de maneira geral indo na mesma direção. Mudanças substanciais também estão em curso no Oriente Médio e na África, mesmo se o quadro geral dessas regiões é mais diversificado. E a América Latina se encontra em algum lugar no meio deste processo.

Apesar de seu enorme significado, o avanço da modernidade global até agora passou despercebido na sociologia. O conceito de modernidade global visa lançar luz sobre a constelação de novidades trazida por este avanço, bem como sobre algumas das consequências e dos desafios presentes - tanto prática como teoricamente. 
O termo "modernidade global" não é novo, mas é usado principalmente de uma forma genérica e não sistemática. Minha própria conceituação está enraizada na distinção de Talcott Parsons entre o sistema social, o sistema cultural, o sistema de personalidade e o sistema organísmico (ou organismo comportamental). Tanto o modo dedutivo de derivação do sistema quanto as formas substantivas pelas quais Parsons designa seus sistemas devem ser vistos como obsoletos e, portanto, dispensados. Heuristicamente, o esquema não deixa de ser útil, até porque sua capacidade de capturar a complexidade da modernidade é inigualável por qualquer das alternativas atualmente oferecidas na literatura das ciências sociais.

Com base nesse esquema de mapeamento do campo, obtém-se um conceito de mudança quadridimensional, com processos de modernização envolvendo transformações fundamentais em cada dimensão, todas inter-relacionadas. Graficamente, isso pode ser descrito da seguinte forma:

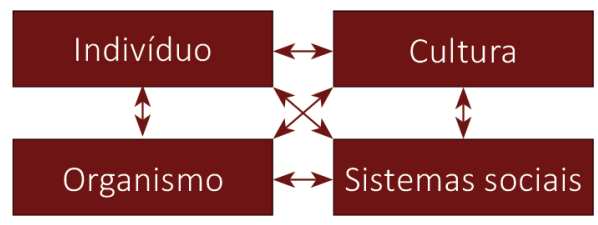

Em suma, os seguintes desenvolvimentos estruturais (discutidos em maior profundidade em um livro recente, ver Schmidt, 2014) se propõem a descrever a condição moderna:

1. Modernização da sociedade (= um dos vários sistemas sociais): diferenciação funcional.

2. Modernização da cultura: racionalização, crescimento da reflexividade/compreensão da maleabilidade dos assuntos humanos e ambientes, generalização de valor.

3. Modernização do indivíduo: surgimento de Eus ativistas, múltiplos e reflexivos; aumento da individuação; capacidades cognitivas melhoradas.

4. Modernização do organismo: disciplina e aperfeiçoamento do corpo humano.

O propósito do esquema é esboçar uma estrutura analítica que demarque o conjunto de fenômenos que devem ser minimamente levados em consideração, se quisermos chegar a uma compreensão minimamente significativa da modernida- 
de (global). Sua utilidade deve, em última instância, provar-se empiricamente. O pressuposto subjacente é que os padrões considerados como características estruturais do moderno não estão confinados a qualquer localização particular. Em vez disso, refletem desenvolvimentos universais que se materializam onde quer que arranjos modernos se enraízem. Isso pode se dar em graus diferentes, em diferentes dimensões, de formas diferentes e em diferentes combinações dos vários elementos. Mas a ausência total de qualquer desses elementos refutaria a validade do esquema.

Como tentei mostrar, o avanço da modernidade global é um incidente razoavelmente recente. Este argumento está em desacordo com outras propostas que postulam um avanço muito anterior, tal como é a formulação de Wallerstein sobre o surgimento do sistema capitalista mundial entre os séculos XV e XVI e, já que para ele o capitalismo moderno é o aspecto mais importante da modernidade, tudo o mais que associamos à condição moderna deriva dele, em maior ou menor grau, e não passa de epifenômeno dessa transformação fundamental. Essencialmente, Immanuel Wallerstein se concentra, portanto, na economia, argumentando que a transição para a modernidade envolve a transição de um sistema de impérios determinado politicamente para uma economia mundial capitalista, que, a partir de então, toma a frente e domina o sistema político (Wallerstein, 1974: 2004). Essa visão é rejeitada pelos defensores de um segundo posicionamento a considerar a ordem verdadeiramente internacional nascida no século XIX, caracterizada como a chave para o surgimento da modernidade global (Buzan \& Lawson, 2015). Esses autores continuam a tratar a política como sistema social condutor e, embora concebam a existência de um sistema econômico internacional desde as viagens de descoberta europeias, consideram a mudança de um poder policêntrico politicamente determinado para uma ordem de inter-relações mundiais ocidentocêntricas, permanentes e multidimensionais, ainda que assimétricas, como o elemento mais essencial na transição para a modernidade global. Uma terceira posição trata do colapso do coIonialismo após a Segunda Guerra Mundial e a subsequente ascensão global do Estado-nação como o ponto de virada histórica mundial, que introduz a modernidade global. Essa é a posição da escola da entidade política mundial neoinstitucionalista de John Meyer e de seus seguidores (Meyer et alii, 1997). Finalmente, minha própria posição empurra o avanço da modernidade global ainda mais para o presente, entre os anos 1970, ou mesmo 1980, e hoje.

Eu acredito que as quatro posições podem estar bem fundamentadas, então nenhuma deve ser imediatamente dispensada. Como este não é o lugar para a defesa plena de minha posição, limito-me a algumas notas de apoio. Durante uma conversa que tivemos em agosto de 2014, o cientista político Jing Huang sugeriu os seguin- 
tes números como indicadores aproximados do grau em que a revolução moderna havia penetrado e mobilizado a população humana em diferentes momentos: 40 milhões de pessoas em 1850; 400 milhões de pessoas em 1950; e 4 bilhões de pessoas hoje. Com a população mundial em 1,2 bilhão em 1850, 40 milhões de pessoas representavam menos de $4 \%$ do total. Em 1950, esse total havia crescido para 2,5 bilhões, de modo que o aumento de dez vezes no número de pessoas agora incluídas no sistema moderno refletia uma parcela de aproximadamente um sexto da população mundial. Essa parcela aumenta para quase dois terços dos atuais 7 bilhões de habitantes do mundo, se, de fato, cerca de 4 bilhões de pessoas têm suas vidas moldadas fundamentalmente por arranjos modernos.

Ao supor que esses números sejam confiáveis - como muito provavelmente o são -, dão-nos uma ideia do grau em que a população mundial foi incluída nos sistemas mais importantes da sociedade moderna em diferentes pontos no tempo. A escola de pensamento da diferenciação do social - cujas premissas compartilho amplamente - trata a diferenciação da sociedade em uma multiplicidade de sistemas sociais encarregados de funções distintas como a característica estrutural fundamental da modernidade societária. A diferenciação funcional é um processo demorado, que leva séculos para se completar e, pelo menos formalmente, abre todos os subsistemas da sociedade ao acesso de toda a população, com base em critérios técnicos e de domínio específico. Esses critérios substituem a descendência e outros fatores determinam o status social e a posição das pessoas nas sociedades estratificadas e hierarquicamente diferenciadas que precedem a sociedade moderna. Embora inicialmente confinada a alguns locais e grupos sociais de elite em seu berço europeu, a diferenciação funcional acabou por dar origem a uma sociedade mundial cujo alcance transcende todas as fronteiras previamente existentes, incluindo as fronteiras políticas do Estado-nação, que nacionalizaram quase todos os aspectos da sociedade no século XIX (Sassen, 2007) antes de dar lugar às globalizações do final do século XX. Entretanto, embora as operações dos sistemas visem principalmente todo o planeta desde o início (como Marx argumentou enfaticamente em defesa da economia), a esmagadora maioria da humanidade continuou excluída das conquistas evolucionárias da modernidade até recentemente. E a exclusão social em grande escala é incompatível com a lógica da diferenciação funcional, afirma Niklas Luhmann (2004), o principal expoente da teoria da diferenciação contemporânea. Uma sociedade funcionalmente diferenciada - portanto plenamente moderna - não pode emergir em escala global até que pelo menos a maioria da população mundial seja incluída, ainda que modestamente, em seus principais sistemas. Assim como a estimativa de Huang e os dados relatados anteriormente sugerem, essa é uma condição que o mundo não começou a conhecer antes da virada do milênio. 
Segundo Luhmann (2012), uma sociedade moderna plenamente desenvolvida não é apenas uma sociedade funcionalmente diferenciada, mas também uma sociedade que abrange o mundo, o que significa que, após o avanço da modernidade global, não faz mais sentido pensar em sociedade no plural. Em vez disso, uma sociedade mundial singular substitui a multiplicidade de sociedades que existiram ao longo da história. Isso tem implicações substanciais para a análise social. Uma tal implicação é a necessidade de descartar o nacionalismo metodológico que iguala a sociedade ao Estado-nação e que tem guiado as ciências sociais desde seu início. Não posso entrar em detalhes aqui, mas espero que o precedente mostre que olhar para o mundo como um todo e analisar dados em escala mundial, em vez de dados específicos de cada país, pode produzir percepções interessantes. Outro ponto forte da teoria da diferenciação ao estilo de Luhmann é que, ao direcionar o olhar analítico para uma multiplicidade de subsistemas sociais em vez de apenas um ou dois (tipicamente a economia ou a política), evita-se os reducionismos que assaltam a análise sociológica até os dias atuais. A inclusão que fiz de dados dos sistemas educacional e científico, embora longe de fornecer uma visão abrangente da sociedade mundial, é impulsionada em parte por essa preocupação.

\section{IV}

Permitam-me agora concluir com algumas notas, mesmo se incompletas, sobre as consequências do avanço da modernidade global. Uma dessas consequências começou recentemente a atrair o interesse crescente de várias disciplinas das ciências sociais: a mudança dos centros de poder da modernidade do Ocidente para o Oriente e outras partes do mundo. Como mencionado acima, grande parte da recente mudança está concentrada na Ásia, especialmente no leste da Ásia. A limitação de espaço impede uma análise completa, portanto um breve exame sobre a economia deve bastar. Enquanto as rendas per capita cresceram 62\% nos Estados Unidos e 74\% no Reino Unido entre 1980 e 2009, estas taxas, por mais notáveis que sejam, são mais baixas do que as exibidas pela Índia (+ 230\%), Coreia do Sul (+ 360\%), região Ásia-Pacífico (+ 594\%) e China (+ 1.083\%) (Eckes, 2011: 9). Quando o desempenho inter-regional varia consideravelmente durante longos períodos de tempo, isso altera o peso relativo das regiões do mundo. A mudança desse tipo talvez mais determinante na história econômica moderna foi a ascensão, primeiramente da Europa e depois do Ocidente, após a Revolução Industrial (que hoje é frequentemente chamada de a primeira). Atualmente, estamos em meio a uma mudança igualmente impactante, desta vez do Ocidente para o Oriente. Calculada em termos de paridade de poder de compra (PPC), a participação dos países da União Europeia no produto interno bruto (PIB) mundial era de aproximadamente $31 \%$ em 1980, enquanto a dos Estados Unidos ficava em 25\%, de acordo com o FMI. Ao mesmo tempo, o 
PIB da China representava apenas 2,2\%. Até o ano de 2012, a participação da União Europeia caiu para 19,2\%, a dos Estados Unidos para 19,5\%, enquanto a da China subiu para cerca de 15\% (Global Vision, 2013). Ao observar a Ásia como um todo, a participação do continente no PIB global aumentou de menos de um quarto (23,2\%) em 1990 para 38,8\% 25 anos depois (2014), já superando a produção combinada da União Europeia e dos Estados Unidos em 2012 (38,7 \%). Tais tendências permanecem desde então. Se espera que em 2020, o produto econômico conjunto da União Europeia e dos Estados Unidos deva cair para aproximadamente 30\% do PIB mundial, enquanto o da China tenha um aumento de até 19\%. Cinco anos depois, em 2025, estima-se que a Ásia represente 45\% do PIB mundial e tenha, de longe, a maior fração da classe média mundial (Asian Development Bank, 2011; Deloitte University Press, 2016).

Mudanças similares estão ocorrendo na educação, em ciência e tecnologia e, por fim, mas não menos importante, nos gastos/aumento/capacidade da força militar. Observadas em conjunto, seu impacto é enorme, representando nada menos que um ponto de inflexão na história mundial. Essas mudanças encerram vários séculos de dominância e supremacia globais ocidentais, dando origem a um mundo cada vez mais policêntrico, em que o Ocidente deixa de ser o condutor mais importante, o modelo e o beneficiário da modernização, e é reduzido a um dos vários atores, não mais capaz de determinar as regras do jogo e os parâmetros de mudança para todos.

Uma segunda consequência do avanço da modernidade global é a aceleração e intensificação, sem precedentes, da mudança em todos os aspectos da vida. A aceleração da mudança tem sido uma característica da condição moderna desde o início. Hoje, no entanto, ganha uma nova qualidade. A modernidade contemporânea - mais que qualquer formação social anterior - empoderou um número muito maior de pessoas para desempenhar um papel significativo na transformação de nossa natureza interna e externa. Isto combinado a tecnologias cada vez mais radicais, estoques de capital e conhecimento extraordinariamente expandidos, bem como instituições que valorizam intensamente a inovação, liberta "forças produtivas" (bem como as destrutivas) e engendra potenciais de transformação diferentes de tudo o que tenha ocorrido anteriormente. Além disso, tais forças são extremamente difíceis de controlar por sua natureza altamente descentralizada e descoordenada. A mudança globalmente transformadora agora pode se originar em qualquer lugar e, devido à incomparável conectividade humana, pode ser difundida rapidamente para praticamente todas as partes do mundo, onde novas ideias e tecnologias são instantaneamente captadas e modificadas para criar o trampolim para a próxima rodada de mudanças. A pressão competitiva na economia, mas 
também nas ciências, nas forças armadas e em outros sistemas, acrescenta mais um estímulo à inovação incessante.

Os primeiros sinais de mudança transformadora já aparecem no horizonte. Após os avanços tecnológicos alcançados recentemente em inteligência artificial, robótica, ciência, tecnologia e informação, coleta e processamento de big data, nanotecnologia, impressão 3D e outros campos que estão se lançando no que tem sido rotulado como "Segunda Era da Máquina" (Brynjolfsson \& McAffe, 2014) - uma "Quarta Revolução Industrial" (Schwab, 2016) ou simplesmente "Indústria 4.0" - espera-se que uma mudança altamente disruptiva ocorra na maneira como vivemos, trabaIhamos e nos relacionamos uns com os outros. No curto prazo, isso tem o potencial de transformar as formas do trabalho para bilhões de pessoas em todo o mundo, como um número crescente de estudos já prevê (Frey \& Osborne, 2013; Ford, 2015; Chang \& Huynh, 2016; OECD, 2017).

A longo prazo, uma transformação ainda maior poderia ocorrer, já que as intervenções genético-tecnológicas, neurológicas e outras no corpo humano, impulsionadas pelo desejo de melhorar, ampliar e aperfeiçoar suas funções fisiológicas e cognitivas, podem resultar em avanços substanciais na longevidade e em aumento colossal de inteligência, dando assim origem a uma era pós-humana na qual os humanos tais como os conhecemos são substituídos por uma espécie transumana muito mais poderosa ou por ela marginalizados (ver, por exemplo, Bostrom, 2014; Harari, 2016). Desnecessário afirmar: o surgimento de tal possibilidade constitui enorme desafio. Que os teimemos ou os acolhamos, é melhor que nos preparemos antes que seja tarde.

\section{Referências}

ASIAN DEVELOPMENT BANK. Asia 2050: realizing the Asian Century. Manila (PH): Asian Development Bank, 2011.

BARRO, Robert J.; LEE, Jong-Wha. A new data set of educational attainment in the world, 1950-2010. Journal of Development Economics, v. 104, p. 184-198, 2013.

BORNMANN, Lutz; MUTZ, Rüdiger. Growth rates of modern science: a bibliometric analysis based on the number of publications and cited references. Journal of the Association for Information Science and Technology, v. 66, p. 2215-2222, 2015.

BOSTROM, Nick. Superintelligence. Paths, dangers, strategies. Oxford (UK): Oxford University Press, 2014. 
BRYNJOLFSSON, Eric; MCAFFE, Andrew. The second machine age. Work, progress, and prosperity in a time of brilliant technologies. New York: Norton, 2014.

BUZAN, Barry; LAWSON, George. The global transformation. History, modernity, and the making of international relations. Cambridge (UK): Cambridge University press, 2015.

CHANG, Jae-Hee; HUYNH, Phu. Asean in transformation. The future of jobs at risk of automation. International Labour Office, Bureau of Employers' Activities, Working Paper n. 9. Genève: International Labour Organization, 2016.

CHRISTIAN, David. Maps of time. An introduction to big history. Berkeley (CA): University of California Press, 2004.

CENTRAL INTELLIGENCE AGENCY (CIA). The world factbook, 2017. Disponível em: <https://www.cia.gov/library/publications/the-world-factbook/>. Acessado em: 19 Jun. 2017.

COZZENS, Susan E. The discovery of growth. Statistical glimpses of twentieth-century science. In: KRIGE, John; PESTRE, Dominique (Eds.). Science in the twentieth century, p. 127-142. Amsterdam: Harwood, 1997.

CRONE, Patricia. Pre-industrial society: anatomy of the pre-modern world. Oxford (UK): Basil Blackwell, 1989.

DELOITTE UNIVERSITY PRESS. Packing a mightier punch: asia's economic growhamong global markets continues, 2016. Disponível em: <http://dupress.com/articles/ asia-pacific-economic-outlook-q1-2016-asia-economic-growth-continues/>.

EASTERLIN, Richard A. The worldwide standard of living since 1800. Journal of EConomic Perspectives, v. 44, p. 7-26, 2000.

ECKES, Alfred E., Jr. The contemporary global economy. A history since 1980. Chichester (UK): Wiley-Blackwell, 2011.

EUROSTAT. The EU in the world - economy and finance, 2015. Disponível em: <http://ec.europa.eu/eurostat/statistics-explained/index.php/The_EU_in_the_ world_-_economy_and_finance>.

FOOD AND AGRICULTURAL ORGANIZATION OF THE UNITED NATIONS (FAO). World-wide estimates and projections of the agricultural population and labour force 1950-2010. Roma: FAO, 2000.

FIREBAUGH, Glenn. The new geography of global income inequality. Cambridge (MA): Harvard University Press, 2003. 
FORD, Martin. Rise of the robots. Technology and the threat of a jobless future. New York: Basic Books, 2015.

FREY, Carl B.; OSBORNE, Michael A. The future of employment: how susceptible are jobs to computerisation? Oxford (UK): Oxford University Press, 2013. Disponível em: $<$ http://www.oxfordmartin.ox.ac.uk/downloads/academic/The_Future_of_Employment.pdf>.

GLOBAL VISION. EU-28 is no longer the world's largest economy, 2013. Disponivel em: <http://www.global-vision.net/blogging-brussels--beyond/eu-28-is-no-longer-the-worlds-largest-economy>.

HARARI, Yuval Noah. Homo Deus. A brief history of tomorrow. New York: Harper Collins, 2016.

HU, Albert G. Z.; ZHANG, Peng; ZHAO, Lijing. China as number one? evidence from china's most recent patenting surge. Journal of Development Economics, v. 124, p. 107-119, 2017.

KHARAS, Homi. The emerging middle class in developing countries. OECD Development Centre Working Paper n. 285. Paris: OECD, 2010.

KUMAR, Krishan. Modernization and industrialization. in: WATERS, Malcolm (Ed.). Modernity. Critical concepts, v. 1: "Modernization", p. 72-94. London: Routledge, 1999.

LUHMANN, Niklas. Theory of society. v. 1. Stanford (CA): Stanford University Press, 2012.

Law as a social system. Oxford (UK): Oxford University Press, 2004.

MADDISON, Angus. The world economy: a millennial perspective. Paris: OECD, 2001.

MEYER, John W.; BOLI, John; THOMAS, George M.; RAMIREZ, Francisco O. World Society and the Nation State. American Journal of Sociology, v. 103, p. 144-182, 1997.

NATIONAL COUNCIL OF INTELLIGENCE. Global trends 2025: a transformed world. Washington (DC): Government Printing Office, 2008.

NATIONAL SCIENCE BOARD. Science and engineering indicators 2016, 2016. Disponível em: <https://www.nsf.gov/statistics/2016/nsb20161/\#/>.

ORGANISATION FOR ECONOMIC CO-OPERATION AND DEVELOPMENT (OECD. OECD employment outlook 2017. Paris: OECD, 2017. 
PECK, Jamie. Offshore. Exploring the worlds of global outsourcing. Oxford (UK): Oxford University Press, 2017.

PRICE, Derek J. De Solla. Little science, big science. New York: Columbia University Press, 1963.

ROSER, Max; ORTIZ-OSPINA, Estaban. Global extreme poverty. Our world in data, 2013. Disponível em: <https://ourworldindata.org/extreme-poverty/>. Acessado em: 15 Jun. 2017.

SASSEN, Saskia. A sociology of globalization. New York: Norton, 2007.

SCHMIDT, Volker H. Global modernity. A conceptual sketch. Basingstoke (UK): Palgrave Macmillan, 2014.

- One world, one modernity. In: SCHMIDT, Volker H. (Ed.). Modernity at the beginning of the 21st century, p. 205-228. Newcastle (UK): Cambridge Scholars Publishing, 2007.

SCHOFER, Evan; MEYER, John W. The worlwide expansion of higher education in the twentieth century. American Sociological Review, v. 70, p. 898-920, 2005.

SCHWAB, Klaus: The Fourth Industrial Revolution. Genève: World Economic Forum, 2016.

STATISTISCHES BUNDESAMT. Bildung und Kultur. Personal an Hochschulen. Wiesbaden (DE): Statistisches Bundesamt, 2016.

UNITED NATIONS DEVELOPMENT PROGRAMME (UNDP). Human development report 2014. Sustaining human progress: reducing vulnerabilities and building resilience. New York: UNDP, 2014.

UNITED NATIONS EDUCATIONAL, SCIENTIFIC AND CULTURAL ORGANIZATION (UNESCO). Unesco science report. Towards 2030. Paris: Unesco, 2015.

- Higher education in Asia: expanding out, expanding up. Montréal (CA): The Unesco Institute for Statistics, 2014.

- The state of science and technology in the world, 1996-97. Montreal: The Unesco Institute for Statistics, 2001.

UNITED NATIONS (UN). World urbanization prospects. Revision 2014. New York: UN, 2014. 
WALLERSTEIN, Immanuel. World-systems analysis. An introduction. Durham (NC): Duke University Press, 2004.

- The modern world system I. Capitalist agriculture and the origins of the European world-economy in the sixteenth century. New York: Academic Press, 1974.

WORLD INTELLECTUAL PROPERTY INDICATORS (WIPO). World Intellectual Property Indicators 2014. Genève: Wipo, 2014.

WORLD BANK. Science and technical journal articles, 2017. Disponível em: <http:// data.worldbank.org/indicator/IP.JRN.ARTC.SC. . . Acessado em: 24 Mar. 2017. 